\title{
Erratum to: Modeling a smooth elastic-inelastic transition with a strongly objective numerical integrator needing no iteration
}

\author{
M. Hollenstein · M. Jabareen · M. B. Rubin
}

Published online: 29 November 2014

(C) Springer-Verlag Berlin Heidelberg 2014

\section{Erratum to: Comput Mech (2013) 52:649-667 DOI 10.1007/s00466-013-0838-7}

At the time of publication of this paper, the authors were unaware of the small deformation models in $[1,2]$ and the large deformation model in [3]. Lubliner et al. [2] generalized previous work by the first author and developed a rateindependent overstress model with a smooth elastic-inelastic transition for which the inelastic deformation rate depends linearly on the stress rate. Their model included both kinematic and isotropic hardening and the comparison with the present model can only be made for the isotropic hardening case. The model in [2] was generalized for large deformations in [3] but the function $\mathrm{h}$ used in this generalization limited attention to sharp elastic-inelastic transitions. Einav [1] generalized previous hypo-plastic and hyper-plastic models which produce rate-independent smooth stress-strain curves with no elastic range and for which the inelastic deformation rate depends linearly on the total strain rate. The model in this paper can be considered as a unifying generalization to large deformations of the models in [1-3] and a viscoplastic overstress model [4] since it models both rate-independent and

The online version of the original article can be found under doi:10.1007/s00466-013-0838-7.

M. Hollenstein · M. B. Rubin ( $₫)$

Faculty of Mechanical Engineering, Technion-Israel Institute

of Technology, 32000 Haifa, Israel

e-mail: mbrubin@tx.technion.ac.il

M. Hollenstein

e-mail: marc.hollenstein@alumni.ethz.ch

M. Jabareen

Faculty of Civil and Environmental Engineering,

Technion-Israel Institute of Technology, 32000 Haifa, Israel

e-mail: cvjmah@techunix.technion.ac.il rate-dependent materials exhibiting: smooth elastic-inelastic transitions with finite elastic ranges and smooth stress-strain curves with no finite elastic ranges.

Acknowledgments The authors would like to thank A. Ibrahimbegovic and I. Einav for informing us of the references [2] and [1], respectively.

\section{References}

1. Einav I (2012) The unification of hypoplastic and elasto-plastic theories. Int J Solids Struct 49:1305-1315

2. Lubliner L, Taylor RL, Auricchio F (1993) A new model of generalized plasticity and its numerical implementation. Int J Solids Struct 30:3171-3184

3. Panoskaltsis VP, Polymenakos LC, Soldatos D (2008) On large deformation generalized plasticity. J Mech Mater Struct 3:441-457

4. Perzyna $P(1963)$ The constitutive equations for rate sensitive plastic materials. Q. Appl. Math. 20:321-332 